\title{
Extrastriate projections in human optic radiation revealed by fMRI-informed tractography
}

\author{
Ivan Alvarez • D. Samuel Schwarzkopf • \\ Chris A. Clark
}

Received: 20 December 2013/ Accepted: 14 May 2014/Published online: 6 June 2014

(C) The Author(s) 2014. This article is published with open access at Springerlink.com

\begin{abstract}
The human optic radiation (OR) is the main pathway for conveying visual input to occipital cortex, but it is unclear whether it projects beyond primary visual cortex (V1). In this study, we used functional MRI mapping to delineate early visual areas in 30 healthy volunteers and determined the termination area of the OR as reconstructed with diffusion tractography. Direct thalamo-cortical projections to areas $\mathrm{V} 2$ and V3 were found in all hemispheres tested, with a distinct anatomical arrangement of superior-inferior fiber placement for dorsal and ventral projections, respectively, and a medio-lateral nesting arrangement for projections to V1, V2 and V3. Finally, segment-specific microstructure was examined, revealing sub-fascicular information. This is to date the first in vivo demonstration of direct extrastriate projections of the OR in humans.
\end{abstract}

Keywords Optic radiation - Visual pathways . Extrastriate · fMRI · Diffusion tensor imaging · Tractography

I. Alvarez $(\bowtie) \cdot$ C. A. Clark

Institute of Child Health, University College London,

London WC1N 1EH, UK

e-mail: ivan.alvarez.11@ucl.ac.uk

D. S. Schwarzkopf

Cognitive, Perceptual and Brain Sciences, University College

London, London WC1H 0AP, UK

\section{Introduction}

The optic radiation (OR) is the most prominent white matter relay in the visual system, conveying information from the lateral geniculate nucleus (LGN) to the occipital cortex. There has been considerable interest in the neuroanatomy and organization of the OR, as it remains an area of specific risk in resection of the anterior temporal lobe (Sincoff et al. 2004). Such resections can lead to unintended damage to the OR, leading to visual field defects in $48-100 \%$ of patients undergoing anterior temporal lobe resections (Winston et al. 2011).

The human OR has been classically described in post-mortem dissections (e.g., Ebeling and Reulen 1988) and histological slices (Wahler-Lück et al. 1991; Bürgel et al. 1999), and its anatomical course is well documented. Originating at the LGN, the OR is divided into three distinct fiber bundles; anterior, central and posterior. The anterior bundle runs anteriorly above the temporal horn of the lateral ventricle, forming Meyer's loop over the temporal lobe before turning posteriorly. The central bundle projects laterally from thalamus across the roof of the temporal horn. The posterior bundle runs directly posterior from LGN and joins the other bundles, with all three blending at the level of the sagittal stratum (Peuskens et al. 2004). The merged fibers then run posteriorly and terminate in the vicinity of the calcarine sulcus (Rubino et al. 2005). These three anatomical bundles convey information of different portions of the visual field, with the anterior, central and posterior bundle representing the upper, central and lower visual field, respectively (Peltier et al. 2006; Pujari et al. 2008; Párraga et al. 2012).

More recently, the OR has also been visualized in vivo using diffusion MRI tractography (Basser et al. 2000). 
Early reports implementing diffusion tensor methods reconstructed the bundle division of the OR (Catani et al. 2003), while more recent work using probabilistic approaches showed reliable estimation of the course of the OR in agreement with post-mortem descriptions (Sherbondy et al. 2008; Clatworthy et al. 2010; Hofer et al. 2010). Tractography reconstructions of the OR have proven useful beyond providing an anatomical description, particularly in presurgical planning (Powell et al. 2005; Yogarajah et al. 2009) but also in intra-operative image guidance (Daga et al. 2012; Winston et al. 2012), and for studying microstructural changes in disease (Bridge et al. 2009; El-Rafei et al. 2011; Kolbe et al. 2011; Groppo et al. 2012).

Despite the growing interest, little work has been conducted exploring the functional significance of white matter fibers-i.e., what representations do distinct fiber populations carry from one region to another (but see Dougherty et al. 2005; Kim et al. 2006; Saenz et al. 2010). In the case of the OR, spatial visual information is highly segregated; both the LGN (Chen et al. 1999; Schneider et al. 2004) and occipital cortex (Tootell et al. 1998; Wandell et al. 2011) follow a retinotopic organization with direct connections between homologous representations of visual space (e.g., Reid and Alonso 1995). As a consequence, damage to specific portions of the OR leads to blindness in a specific segment of the visual field, such as hemianopsia or quadrantanopsia (e.g., Barton et al. 2005).

While the functional segregation of visual field quadrants in OR bundles is well established, it remains unclear whether these projections reach beyond primary visual cortex (V1). In animal models such as the cat (Garey and Powell 1971; Maciewicz 1975) and flying fox (Manger and Rosa 2005), LGN projections to areas V2 and V3 are well established in tracer studies. In the macaque, anterograde and retrograde tracer evidence suggest a direct projection to extrastriate areas (Yukie and Iwai 1981), specifically areas 18 and 19 (Benevento and Yoshida 1981; Fries 1981). While this hypothesis has been contested by negative findings (Yoshida and Benevento 1981; Benevento and Standage 1982), later experiments using fluorescent dyes support the presence of a direct LGN to V2 projection (Bullier and Kennedy 1983; Kennedy and Bullier 1985). The intriguing possibility of a direct extrastriate projection in the macaque, the predominant model species used to inform our understanding of visual system architecture, begets the question of whether this organization also exists in the human. This study presents the first in vivo evidence for direct OR projections to regions $\mathrm{V} 2$ and $\mathrm{V} 3$ in human occipital cortex using diffusion MRI tractography.

\section{Methods}

Participants

Thirty healthy adults (12 males, age range $21-35$ ) took part in the study. All participants had normal or corrected-tonormal visual acuity and provided written informed consent. This study was approved by the UCL Research Ethics Committee (London, UK).

\section{Behavioral measures}

In order to assess hand dominance, we used the Edinburgh Handedness Inventory, a 10-item self-report questionnaire probing the lateralization of hand use in everyday tasks (Oldfield 1971). Hand dominance scores were rated on a scale between -100 and 100 , with negative numbers indicating a tendency toward left-handedness and positive numbers a tendency toward right-handedness. Participants scored an average of $84.76 \pm 6.54$ SEM, with 27 participants identified as right-handers (scoring over 50) and 1 participant identified as left-handed (scoring under -50 ).

Ocular dominance was assessed using the hole-in-card test, also known as the Dolman method (Seijas et al. 2007). The participants held a $300 \times 220 \mathrm{~mm}$ card at arms length and view a $130 \times 130 \mathrm{~mm}$ object, in this case a painting, at approximately $3 \mathrm{~m}$ distance through the $25 \mathrm{~mm}$ hole on the center of the card. The subject alternatively occluded one eye to establish which eye is aligned with the distant object. The eye that upon occlusion causes the image of the target to disappear is considered the dominant eye. While the hole-in-card shows only moderate agreement with alternative tests of ocular dominance (Kommerell et al. 2003), it has high test-retest reliability (Rice et al. 2008) and was preferred due to its widespread use in the literature. Eye dominance as assessed with the hole-in-card test was a nominal measurement, with 14 left eye dominant and 16 right eye dominant participants. No significant correlation between demographic factors (age, gender) and behavioral measures (hand and eye dominance) were observed (Pearson's $r$, all pairwise comparisons $p>.05$ ).

\section{Visual stimulation}

Stimuli were generated in MATLAB (v8.0, Mathworks Inc., Natick, MA, USA) using Psychtoolbox (v3.0, Brainard 1997; Pelli 1997) and displayed on an MRI-compatible LCD monitor. The participant viewed the monitor through a mirror while laying supine in the bore of the scanner. The visual display subtended $10.40^{\circ}$ of visual angle from fixation.

The stimulus consisted of a $10.40^{\circ}$-radius disk of a dynamic, high-contrast tessellated pseudo-checkerboard 
with a drifting 'ripple-like' pattern that varied across time in spatial frequency and phase. This broadband pattern ensured effective stimulation of visually responsive neurons in visual cortex. Mean luminance of the stimulus was $132.29 \mathrm{~cd} / \mathrm{m}^{2}$.

The stimulus was presented on an equiluminant gray background through a series of sequential apertures in two conditions. First, a mapping stimulus comprised simultaneous 'wedge' and 'ring' apertures - a wedge section of $17.14^{\circ}$ angle rotated clockwise or counter-clockwise along the polar dimension while a ring section expanded or contracted eccentrically, scaling logarithmically between $0.11^{\circ}$ and $4.62^{\circ}$ width span. Apertures changed position every $2.376 \mathrm{~s}$, on the onset of each EPI volume acquired. Both apertures cycled at independent frequencies: 21 and 15 volumes per revolution, respectively. A single run consisted of 10 wedge and 14 ring revolutions, followed by 24 volumes of mean luminance, totaling 238 volumes per run. Two runs were conducted, the first with clockwise wedge and expanding ring motion and the second with counter-clockwise wedge and contracting ring motion.

The second condition consisted of a short photic burst of a circular $10.40^{\circ}$-radius aperture of the same pattern, presented for 1 volume and followed by 14 volumes of equiluminant gray background. Ten runs were conducted, totaling 150 volumes acquired.

Throughout both conditions, a $0.30^{\circ}$ black center-point cross was presented to aid fixation. Participants were instructed to attend and respond when the cross flashed in red, which occurred in a semi-randomized fashion, with 100-150 events per run, each lasting 200 ms. Participant responses were recorded via an electronic response button and monitored to ensure engagement with the task, and therefore consistent eye fixation.

\section{MRI acquisition}

MR images were acquired on a $1.5 \mathrm{~T}$ Avanto MRI system using a 32-channel head coil (Siemens Healthcare, Erlangen, Germany). The experimental session was divided into two consecutive sections taking place on the same day. For the first section only, the bottom elements of the head coil were used, to avoid visual field restrictions. A gradientecho EPI sequence was used ( $\mathrm{TR}=2,376 \mathrm{~ms}$, $\mathrm{TE}=30 \mathrm{~ms}, 33$ ascending slices, $18 \%$ inter-slice gap), with off-axial acquisitions maximizing occipital lobe coverage and effective resolution of $3 \mathrm{~mm}^{3}$ isotropic voxels for 4 subjects and $3.3 \mathrm{~mm}^{3}$ isotropic voxels for 26 subjects. A T1-weighted anatomical image $(\mathrm{TR}=11 \mathrm{~ms}$, $\mathrm{TE}=4.94 \mathrm{~ms}$ resolution $=1 \mathrm{~mm}$ isotropic) was acquired in-plane with the functional images to aid registration, and B0 field maps $\left(\mathrm{TR}=487 \mathrm{~ms}, \quad \mathrm{TE}_{1}=5.28 \mathrm{~ms}\right.$, $\mathrm{TE}_{2}=10.04 \mathrm{~ms}$ ) were acquired to estimate local field inhomogeneities. For the second section, the full head coil arrangement was used and a further anatomical T1weighted volume $(\mathrm{TR}=11 \mathrm{~ms}, \mathrm{TE}=4.94 \mathrm{~ms}$, resolution $=1 \mathrm{~mm}$ isotropic) was acquired to reconstruct the cortical surface, as well as a diffusion-weighted EPI protocol with 60 non-collinear gradient directions at $b=1,000 \mathrm{~s} / \mathrm{mm}^{2}$ and $3 b=0 \mathrm{~s} \mathrm{~mm}{ }^{2}$ reference images ( $\mathrm{TR}=7,300 \mathrm{~ms}, \mathrm{TE}=81 \mathrm{~ms}$, maximum gradient amplitude $=40 \mathrm{mT} / \mathrm{m}^{-1}, \quad 60$ slices, resolution $=2.5 \mathrm{~mm}$ isotropic).

\section{Functional imaging analysis}

T1-weighted anatomical images were processed with FreeSurfer (Dale et al. 1999; Fischl et al. 1999). Volumes underwent automated segmentation to generate gray and white matter boundaries, and the gray matter surface reconstructed to create a smooth two-dimensional representation of cortex.

Functional EPI images were pre-processed in SPM8 (Wellcome Trust Centre for Neuroimaging, http://www.fil. ion.ucl.ac.uk/spm). All images were bias-corrected, realigned to the first image of each run to reduce movement artifacts, unwrapped to correct for field inhomogeneities and slice timing-corrected to reduce variation in the time series introduced by the timing of the slice acquisition. Finally, a two-step co-registration was performed on all functional images, by first registering to the in-plane anatomical T1-weighted image and then registered to the anatomical image acquired with the full 32-channel head coil array, in order to maximize registration precision.

fMRI data from the mapping condition were modeled using a population receptive field ( $\mathrm{pRF}$ ) model using inhouse software in MATLAB. Briefly, model predictions were based on the a priori knowledge of stimulus position during stimulation and the assumption of single Gaussian receptive field (Dumoulin and Wandell 2008). This model prediction was then convolved with the hemodynamic response functions (HRF), individually estimated for each subject by fitting the time series acquired during photic burst stimulation with a double gamma function (Friston et al. 1995). Mapping data were sampled from volume space to the cortical surface reconstructions, spatially smoothed $($ FWHM $=8.3 \mathrm{~mm}$ ) and compared to the model predictions via Pearson's correlation coefficient. Parameter values of the predictive model that yielded the highest correlation were then used as the starting point for fitting the un-smoothed mapping data, using the Nelder-Mead algorithm for unconstrained nonlinear minimization (Lagarias et al. 1998). The winning model outputs were then smoothed across the cortical surface $(\mathrm{FWHM}=5 \mathrm{~mm})$ and projected onto the inflated cortical surface. 
Polar angle estimates derived from the pRF model were displayed on the reconstructed cortical surface to delineate region boundaries between primary visual cortex (V1), areas $\mathrm{V} 2$ and $\mathrm{V} 3$ according to their retinotopic organization (Sereno et al. 1995; DeYoe et al. 1996; Engel et al. 1997). Dorsal and ventral components of these areas were identified, creating six regions per hemisphere sampled: V1d, $\mathrm{V} 1 \mathrm{v}, \mathrm{V} 2 \mathrm{~d}, \mathrm{~V} 2 \mathrm{v}, \mathrm{V} 3 \mathrm{~d}$ and V3v. One additional region, V3A on the anterolateral boundary of dorsal V2, was delineated as a control area. Region definitions were then transformed into volume space to inform tractography.

\section{Diffusion imaging analysis}

Diffusion-weighted images were registered to the first, non-diffusion-weighted volume $(b=0)$ to correct for head motion and eddy-current artifacts using FSL-FDT (Behrens et al. 2003). Data were then analyzed with MRtrix (Tournier et al. 2012) by fitting a diffusion tensor model and calculating fractional anisotropy (FA) and mean diffusivity (MD) maps. FA maps were subsequently thresholded to retain values at or above 0.7 and spatially eroded to create a white matter skeleton mask. Voxels retained in the white matter skeleton were used to estimate the diffusion response function. The full diffusion data were then fitted with a constrained spherical deconvolution model (maximum harmonic order $=8$ ) to produce a multi-fiber model of white matter diffusion at each voxel.

In order to reconstruct the path of the OR, a seed-totarget tractography approach was used. The LGN was manually defined on T1-weighted images for each participant, based on anatomical markers (Fujita et al. 2001) and used as 'seed' regions. Manual delineations aimed at minimizing contributions from neighboring subcortical structures, including the pulvinar and superior colliculus, therefore a conservative definition of the LGN was preferred. Functionally defined visual maps were registered to diffusion space, and a single ROI consisting of voxels belonging to either area V1, V2 or V3 was used as a single target region in probabilistic tractography (step size $=0.2 \mathrm{~mm}$, angular threshold $=1 \mathrm{~mm}$, FA threshold $=0.1$, streamlines $=10,000$ ). This procedure was performed independently for each hemisphere, in each subject. A single target ROI approach was adopted in order to ensure unbiased sampling of the cortical targets, allowing the estimation of the number of streamlines terminating in each target area, rather than defining said number a priori by tracking each target region individually. The direction of tracking, thalamus to occipital, ensured an accurate reconstruction of the highly angular path of the OR at the level of Meyer's loop.

Resulting OR streamlines were displayed in TrackVis (http://www.trackvis.org) and manually constrained to reject anatomically incorrect streamlines. The following heuristic rules were applied; streamlines were rejected if they were (a) crossing to the contralateral hemisphere, (b) projecting superiorly from the LGN, (c) projecting antero-inferiorly, into the temporal pole and (d) projecting parallel to the medial wall of the posterior horn of the lateral ventricle. An average of $67 \%$ of streamlines were rejected [mean number of streamlines rejected $=6,738 \quad(67 \%) \pm 1,341 \quad(13 \%)$ SEM]. This heuristic approach constrained the tractography to the known anatomical course of the OR (Ebeling and Reulen 1988; Párraga et al. 2012), with the aim of increasing the confidence on the virtual reconstruction of the white matter tract and ensuring accurate sampling of microstructural parameters. The procedure was carried out independently for each hemisphere analyzed.

\section{Tract segmentation}

OR tractography results were segmented using two different schemes; one constrained by the representations of upper and lower visual field (visual field-based) and one based on the visual map hierarchy (hierarchy-based). In both cases, a Boolean approach was implemented, using the inclusion (AND) and exclusion (NOT) criteria defined below.

For visual field-based segmentations, streamlines in the OR terminating in dorsal visual areas, representing the lower visual field (V1d, V2d, V3d), were classified as belonging to the 'dorsal' segment, while streamlines terminating in ventral areas, representing the upper visual field $(\mathrm{V} 1 \mathrm{v}, \mathrm{V} 2 \mathrm{v}, \mathrm{V} 3 \mathrm{v})$, were classified as part of the 'ventral' segment. In both cases, streamlines common to both upper and lower visual field representations were excluded.

For hierarchy-based segmentations, streamlines were divided into those projecting exclusively to each of the following cortical areas: V1, V2 and V3. For example, the $\mathrm{V} 1$ projection segment consisted of streamlines projecting to either V1d or V1v, but not V2 or V3 maps. In all cases, dorsal and ventral components were combined.

In summary, two segmentation schemes were applied to the OR in each hemisphere, resulting in five segments: dorsal, ventral, V1, V2 and V3 projections of the OR.

\section{V3A control condition}

An additional condition with a control ROI was carried out in order to estimate the rate of acceptance of invalid streamlines, as an approximation of the false-positive rate in the probabilistic tractography method implemented. Area V3A was chosen as the control ROI, as no direct thalamic projection was expected based on the human or macaque literature. Tractography was performed between 




Fig. 1 Segments of the optic radiation in a representative participant. a Visual field-based segmentation, with dorsal segments following a superior profile, and ventral streamlines populating the more inferior and lateral parts of the tracts. b Hierarchy-based segmentation, with

the LGN and a single target region comprising areas V1, $\mathrm{V} 2, \mathrm{~V} 3$ and V3A. The ratio of streamlines terminating in the control region against those terminating in areas $\mathrm{V} 1$, $\mathrm{V} 2$ or V3 was calculated and used as an estimate of the false-positive rate of the method.

\section{Results}

\section{Anatomy of the OR}

Both striate and extrastriate projections of the OR were identified in all 60 hemispheres tested. Key structural characteristics were observed for both segmentation schemes, which are described in turn.

\section{Visual field-based segmentation}

Dorsal and ventral segments of the OR follow parallel, but distinct anatomical courses (see Fig. 1). The dorsal segment projects laterally from the LGN and maintains a superior profile for the rest of its posterior course toward the occipital lobe. The ventral segment traces a similar anterior path, covering both the anterior and central bundles including Meyer's loop, but then follows an inferior profile spanning the whole anterior-posterior route. From an axial vantage point, the dorsal segment fans out more laterally as it approaches occipital territories, while the ventral segment retains a medial profile. This anatomical arrangement was observed across subjects, with the
$V 1, V 2$ and $V 3$ projections displayed. $V 1$ streamlines form the most medial segment, while $V 2$ and $V 3$ form a nesting pattern dorsolaterally

superior-inferior division of the long segment of the OR being a marked feature visible in all hemispheres tested (see "Appendix 1" for segment renders of all subjects).

\section{Hierarchy-based segmentation}

The arrangement of V1, V2 and V3 segments follows a media-lateral nesting pattern, with the $\mathrm{V} 1$ segment at its center, surrounded by the V2 segment, which in turn is surrounded by V3 (Fig. 2). All three segments follow a similar curved course, with the V1 segment being the most medial part of the tract, and V2 and V3 segments subsequently more lateral (see Fig. 3). It is immediately apparent that the V1 segment is substantially larger than the V2 or V3 segments, which is in agreement with the existing idea of the $\mathrm{OR}$ as a primarily striate projection and also the larger cortical area span of V1 compared to V2 or V3 (Yan et al. 2009). The overall nesting arrangement was again observed in all tested hemispheres (for visitation maps of all subjects, see "Appendix 2").

\section{Variability in OR microstructure}

Microstructural markers were sampled by identifying the voxels traversed by all streamlines belonging to each tract segment and computing the mean value for FA and MD in those voxels for each hemisphere. The number of streamlines retained in each tract segment was also extracted as a relative measure of segment size. 


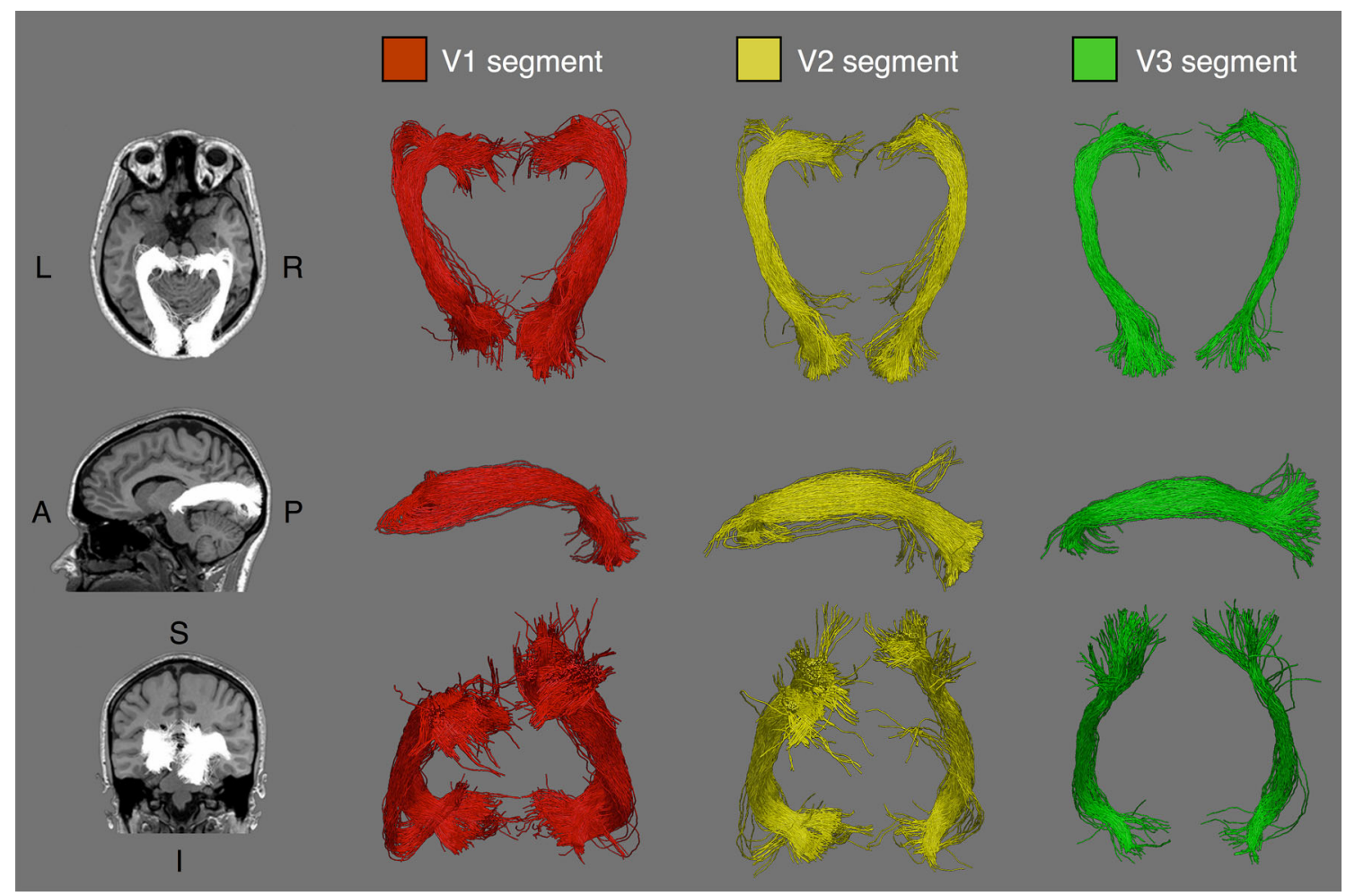

Fig. 2 Hierarchy-based segmentation of the optic radiation in a representative participant. Segments terminating in regions $V 1, V 2$ and $V 3$ are displayed, demonstrating the anatomical course of each segment

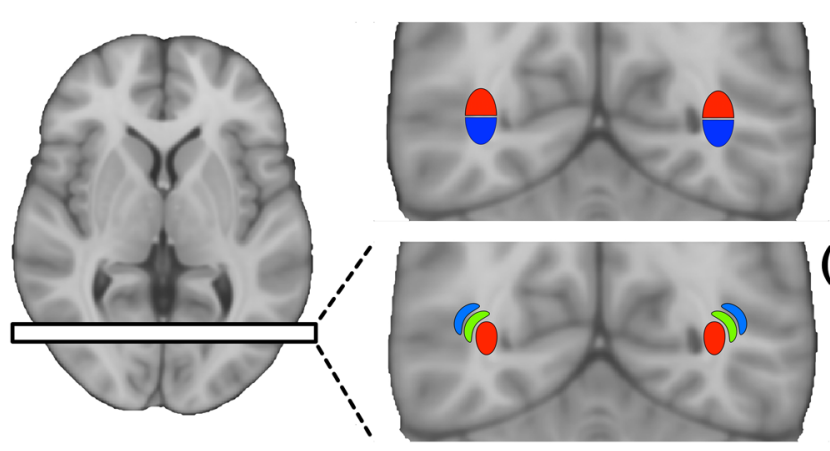

Fig. 3 Visitation maps for optic radiation tractography in a representative participant, visualized on a single coronal slice immediately posterior to the lateral ventricle. a Visual field-based segmentation

In order to test whether microstructural markers varied between visual field-based (dorsal, ventral) and hierarchical-based (V1, V2, V3) segments of the OR, we employed a series of mixed factorial ANOVA tests. Univariate models were preferred due to the exploratory nature of the study. All statistical analyses were carried out in SPSS (v21, IBM SPSS Statistics, IBM Corp., Armonk, New York, USA). (a)


(b)
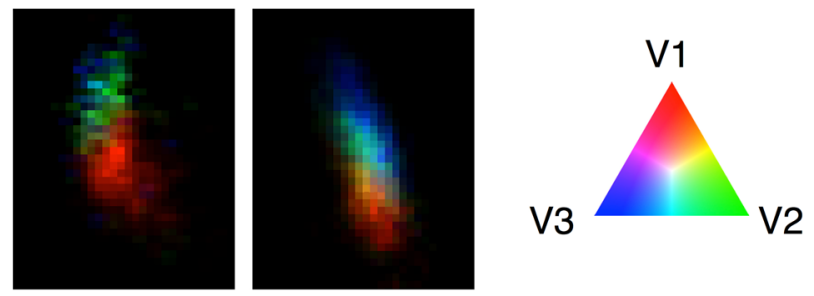

shows the superior-inferior division of streamlines, in red and blue. b Hierarchy-based segmentation discriminates streamlines into dorso-lateral nesting compartments, in red, green and blue

\section{Effect of covariates}

In order to test the effects of hierarchical segmentation scheme on outcome measures, we performed three independent mixed factorial ANOVAs, one for each outcome measure (FA, MD, streamline count). The ANOVA model incorporated hierarchical segmentation scheme and hemisphere as within-subject variables and age, gender, hand 
(a) 100



(b)



Fig. 4 Microstructural values across thirty participants for hierarchybased segmentation $(V 1, V 2, V 3)$ of the optic radiation. Values displayed are a number of streamlines as a percentage of all

dominance and eye dominance as between-subject variables. These tests revealed no significant main effects or interactions (all effects $p>.05$ ) of any between-subject variables, namely age, gender, hand dominance or eye dominance. Therefore, in order to increase statistical power, the between-subject variables were dropped and a restricted model, only incorporating the within-subject variables (segmentation scheme and hemisphere) is presented here.

\section{Visual field-based segmentation}

Three mixed factorial ANOVA models were tested, examining the effect of visual field-based segmentation on the outcome measures described above. A significant effect of segmentation was found on $\mathrm{MD}(F=29.96, d f=1,29$, $p<.001)$ but not FA $(F=1.80, d f=1,29, p=.191)$, with MD being higher in the ventral compared to the dorsal segment. For both MD and FA, a significant effect of hemisphere was observed (MD: $F=102.84, d f=1,29$, $p<.001$; FA: $F=40.96, d f=1,29, p<.001)$, with a
Left optic radiation

Right optic radiation

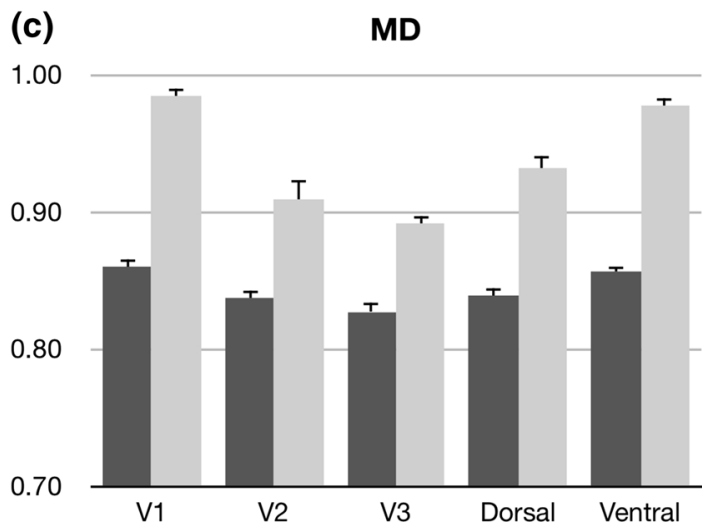

streamlines in the optic radiation, $\mathbf{b}$ mean fractional anisotropy $(F A)$ and c mean diffusivity $(M D)$. Percentage streamlines for each hemisphere total $100 \%$

general tendency toward lower microstructural content in the right hemisphere (reduced FA, increased $\mathrm{MD}$; see Fig. 4).

The increase in mean diffusivity in the ventral segment can be partially attributed to the anatomical course of the two segments described above; with the ventral segment being more proximal to the posterior horn of the lateral ventricle compared to the dorsal segment. It is possible that a partial volume effect with adjacent cerebral spinal fluid influenced the increased diffusivity in the ventral segment. Nevertheless, the lack of concomitant differences in FA between segments suggest other factors, as cerebral spinal fluid contamination typically leads to decreased FA, so microstructural differences between these two segments must be interpreted with caution.

In addition to microstructural markers, a significant effect of segmentation on streamline count was found $(F=25.04, d f=1,29, p<.001)$, with a larger streamline count in dorsal segments compared to ventral segments (Fig. 4), independent of hemisphere $(F=4.09, d f=1,29$, $p=.052)$. 


\section{Hierarchy-based segmentation}

Three mixed factorial ANOVA models were tested, examining the effect of hierarchy-based segmentation and hemisphere on FA, MD and streamline count. A significant effect of segmentation was found on both $\mathrm{MD}(F=92.74$, $d f=2,58, p<.001)$ and FA $(F=4.52, d f=2,58$, $p<.05)$, with significant interactions of segmentation with hemisphere for both microstructural measures (MD: $F=34.78, d f=2,58, p<.001$; FA: $F=48.01, d f=2$, $58, p<.001)$. To further investigate the interaction terms, post hoc paired $t$ tests were performed, revealing differential effects of hemisphere on segment microstructure (Fig. 4). Bonferroni correction for multiple comparisons was applied across all subsequent post hoc tests.

For FA, values decreased with cortical hierarchy in the left hemisphere, with significant step effects between V1 and V2 segments $\left(t=5.96, d f=29, p_{\text {corr }}<.001\right)$ and between V2 and V3 $\left(t=8.78, d f=29, p_{\text {corr }}<.001\right)$. In comparison, the right hemisphere exhibited the opposite effect, with an increase in FA between V1 and V2 segments $\left(t=4.88, d f=29, p_{\text {corr }}<.001\right)$ but no significant difference between $\mathrm{V} 2$ and V3 segments $(t=0.36$, $\left.d f=29, p_{\text {corr }}=.999\right)$.

In the case of MD, a reduction of MD was observed with increasing cortical hierarchy, both in left (V1-V2: $t=6.46, d f=29, p_{\text {corr }}<.001 ; \mathrm{V} 2-\mathrm{V} 3: t=3.23, d f=29$, $\left.p_{\text {corr }}<.05\right)$ and right ORs (V1-V2: $t=8.64, d f=29$, $p_{\text {corr }}<.001$; V2-V3: $\left.t=3.53, d f=29, p_{\text {corr }}<.05\right)$.

Finally, the effect of hierarchy-based segmentation on streamline count was assessed, with a significant effect of segmentation $(F=42.88, d f=2,58, p<.001)$ and no interaction with hemisphere $(F=0.22, d f=2,58$, $p=.800)$. Within each hemisphere, a larger number of streamlines were found in the V1 segment compared to the V2 $\left(t=8.04, d f=59, p_{\text {corr }}<.001\right)$ or V3 segments $\left(t=8.15, d f=59, p_{\text {corr }}<.001\right)$. No significant difference between V2 and V3 segments was found $(t=0.09$, $\left.d f=59, p_{\text {corr }}=.930\right)$.

In order to assess whether the effect of hierarchy-based segmentation on streamline count was driven by the difference in size between target regions, we normalized the streamline counts by dividing each seed-target pair (V1, V2 and V3) by the surface area $\left(\mathrm{mm}^{2}\right)$ of its corresponding target region, independently for each hemisphere (see Fig. 5). A main effect of segmentation was again observed $(F=10.91, d f=2,58, p<.001)$, with no interaction with hemisphere $(F=0.28, d f=2,58, p=.868)$. While the streamline count difference was originally driven by a greater proportion terminating in area V1, following normalization, the streamline count for area V1 was significantly lower when compared to the V2 $(t=4.51$, $\left.d f=59, p_{\text {corr }}<.001\right)$ and V3 projections $(t=5.64$, $\left.d f=59, \quad p_{\text {corr }}<.001\right)$. No significant difference was observed between the V2 and V3 segments $(t=1.07$, $\left.d f=59, p_{\text {corr }}=.864\right)$.

False-positive rate estimation

In order to assess the validity of streamline count estimations, a control condition was introduced where the ratio of streamlines terminating in area V3A against those terminating in regions $\mathrm{V} 1$, V2 or $\mathrm{V} 3$ was used as an estimate of the false-positive rate of the tractography method. Under this condition, a significantly smaller proportion of streamlines terminated in the control site [Mean $=85$ $(4 \%) \pm 24(1 \%)$ SEM] compared to either area V2 [Mean $=427(20 \%) \pm 76(28 \%)$ SEM; pairwise $t$ test, $t=6.33, d f=59, \quad p<.001] \quad$ or $\mathrm{V} 3 \quad[$ Mean $=423$ $(20 \%) \pm 62(23 \%)$ SEM; pairwise $t$ test, $t=8.86$, $d f=59, p<.001]$. Furthermore, when the same data were normalized based on the surface area $\left(\mathrm{mm}^{2}\right)$ of the corresponding target area, the hypothesized extrastriate projections to V2 $(t=7.18, d f=59, p<.001)$ and V3 $(t=9.80, d f=59, \quad p<.001)$ retained a significantly greater proportion of streamlines when compared to the control area. This indicates that projections to areas V2 and V3 were not only larger than to the control site in real terms, as estimated by streamline count, but also in relative terms, while accounting for the cortical extent of the target region (see Fig. 5).

\section{Discussion}

In this work, we identified extrastriate projections of the OR to areas V2 and V3 of occipital cortex in humans, in agreement with the macaque literature (Yukie and Iwai 1981; Benevento and Yoshida 1981; Fries 1981; Bullier and Kennedy 1983; Kennedy and Bullier 1985) and highlighting the anatomical homology of the retino-geniculatestriate pathway across primate species.

The OR projections to dorsal and ventral representation in occipital cortex follow anatomically distinct courses, with a predominance of upper visual field representation in the anterior bundle of the OR, in agreement with human dissection data (Ebeling and Reulen 1988; Wahler-Lück et al. 1991; Peuskens et al. 2004; Peltier et al. 2006) and a superiorinferior divide along the anterior-posterior segment. Similarly, the hierarchical projection segments of the OR follow 
(a) $100 \%$

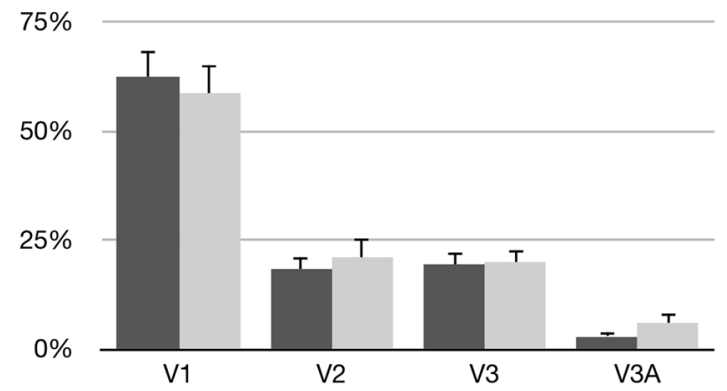

(b) $100 \%$

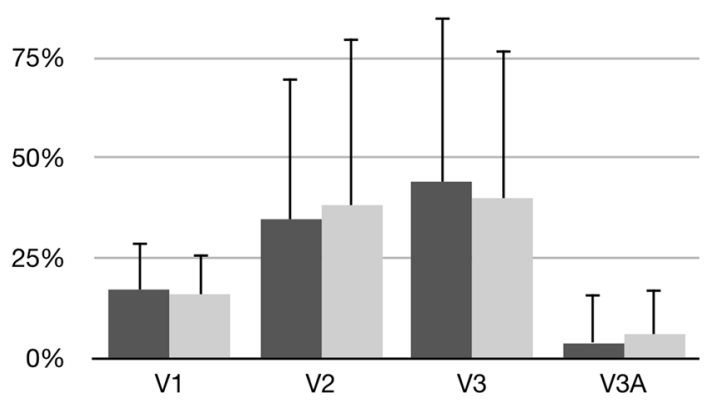

Fig. 5 Streamline counts for three hierarchy-based segmentations of the optic radiation $(V 1, V 2, V 3)$ and one control region, $V 3 A$. Values displayed are a number of streamlines as a percentage of all streamlines in the optic radiation, $\mathbf{b}$ number of streamlines normalized

anatomically segregated courses, with a medio-lateral nesting pattern. Both of these findings are consistent with the proposed hypothesis of distinct bundles connecting retinotopic LGN locations with a matching retinotopic representation in occipital cortex.

\section{Relative projection size}

A larger V1 projection was observed bilaterally, when compared to connections with V2 and V3, as estimated by the relative number of streamlines terminating in each visual area. This finding is consistent with the idea of the OR as a primarily striate projection, but also with the overall larger cortical area representing V1, when compared to its neighboring maps V2 and V3 (Yan et al. 2009). We confirmed that cortical area size played an important role in this regard by examining the effects of normalization by target surface area on the observed streamline counts. The direction of the effect was in fact reversed, with areas V2 and V3 displaying proportionally larger number of streamlines terminating at those sites, when compared to the V1 projection. As evidenced here,
Left hemisphere

Right hemisphere

(c)

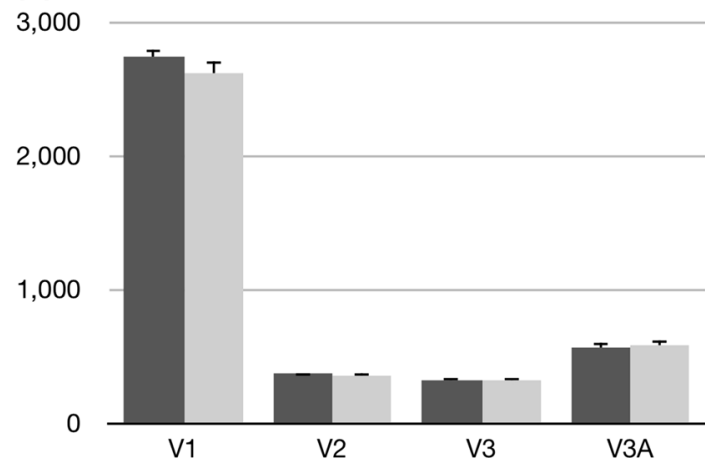

by the surface area of the target region and $\mathbf{c}$ the surface area of the target region, in $\mathrm{mm}^{2}$. Percentage streamlines for each hemisphere total $100 \%$

projections to extrastriate areas V2 and V3 in fact account for a greater proportion of all OR streamlines per $\mathrm{mm}^{2}$ of cortical target zones.

Additionally, in order to provide an estimate of falsepositive rate of the method implemented, we included a control site, V3A, to analyze the likelihood of generating streamlines to a cortical site where no direct thalamic projections were expected. In comparison to the control condition, projections to areas V2 and V3 were shown to be significantly larger, both in terms of number of streamlines reaching the target, and also when accounting for the surface area of the target zone. These results increase confidence on the $\mathrm{V} 2$ and $\mathrm{V} 3$ projections having a true anatomical basis and are unlikely to be false-positive connections arising as an artifact of the probabilistic nature of the tractography algorithm as indicated by the control region V3A that was examined.

\section{Microstructural findings}

A hemispheric lateralization effect of OR microstructure was observed in all subjects tested, with lower FA and 
higher MD in the right, compared to the left OR (illustrated in Fig. 4). This result is consistent with the histological literature, where both the cortical (Bürgel et al. 1999) and the white matter area (Weinberger et al. 1982) of the left occipital region are found to be larger in the left than the right hemisphere. This hemispheric asymmetry would cause a detectable difference in microstructural measures, as larger, and more densely packed axonal bundles in the left hemisphere lead to increased FA and reduced MD, as reproduced in this study.

Perhaps more intriguing is the pattern of FA measured in hierarchy-based segmentations, where the left hemisphere displayed increased FA from V1 to V2 and V3, while the right hemisphere displayed and inverse pattern. An overall large tract volume in the left hemisphere, as previously noted, does not fully account for this observation and instead, it points toward a larger affordance of volume in the left OR sustaining a microstructurally robust V1 projection, while this affordance may be diminished in the smaller right OR. In real terms, the differential pattern of FA values across hierarchy-based segments of the OR is a small effect, as the variability between tract segments does not exceed FA $<0.05$ in any single hemisphere. Nevertheless, such a pattern hints toward potential structural differences in white matter organization beyond binary hemispheric effects, and these findings suggest such variability may apply to specific segments of white matter pathways in isolation.

\section{Role of extrastriate projections}

The existence of direct thalamo-cortical projections to extrastriate regions is perhaps not surprising; the visual system can be conceptualized as a hierarchical structure with a high degree of modularity, with distinct feature processing occurring in parallel and integrated to form a representation of visual information. As such, this system requires the provision of input; principally, but not exclusively, via the retino-geniculate pathway to multiple early regions for parallel processing and feed-forward to higher areas in occipital cortex and beyond. In addition, a large amount of connections along the OR are feedback connections, providing a bidirectional bridge between subcortical and cortical processing of visual information (Van Essen and Maunsell 1983; Felleman and Van Essen 1991).
While it is not possible to distinguish feed-forward from feed-back projections with tractography techniques, direct extrastriate projections in human OR are likely to support both types of connection in V1 and at least feed-forward connectivity to extrastriate projections, in agreement with the structural arrangement seen in the macaque (Bullier and Kennedy 1983; Kennedy and Bullier 1985). In its entirety, the OR forms a substantial component in wider visual system, affording both parallel, bidirectional connections to multiple levels of cortical and sub-cortical areas involved in the processing of visual input.

\section{Conclusion}

While typically described as a striate projection, the OR is hypothesized to connect the thalamus with extrastriate visual areas and in this study, we have identified and described direct OR projections to areas V2 and V3, in addition to striate cortex, $\mathrm{V} 1$ in humans in vivo. The arrangement of tract segments reveals anatomical segregation of upper and lower visual field representations into dorsal and ventral segments, as well as distinct white matter segments connecting to areas V1, V2 and V3 following a medio-lateral nesting pattern identifiable in single participants. Finally, microstructural differences between segments of the OR point to finer lateralization effects than previously considered, with significant differences in FA for V1 segments but not for extrastriate projections. Taken together, these findings point to a more nuanced functional arrangement of the human OR than previously considered, with the combination of functional and diffusion MRI allowing the tracing of specific eloquent segments of the white matter pathway into extrastriate regions.

Acknowledgments This work was supported by a University College London Grand Challenges Studentship.

Open Access This article is distributed under the terms of the Creative Commons Attribution License which permits any use, distribution, and reproduction in any medium, provided the original author(s) and the source are credited.

\section{Appendix 1}

See Fig. 6. 


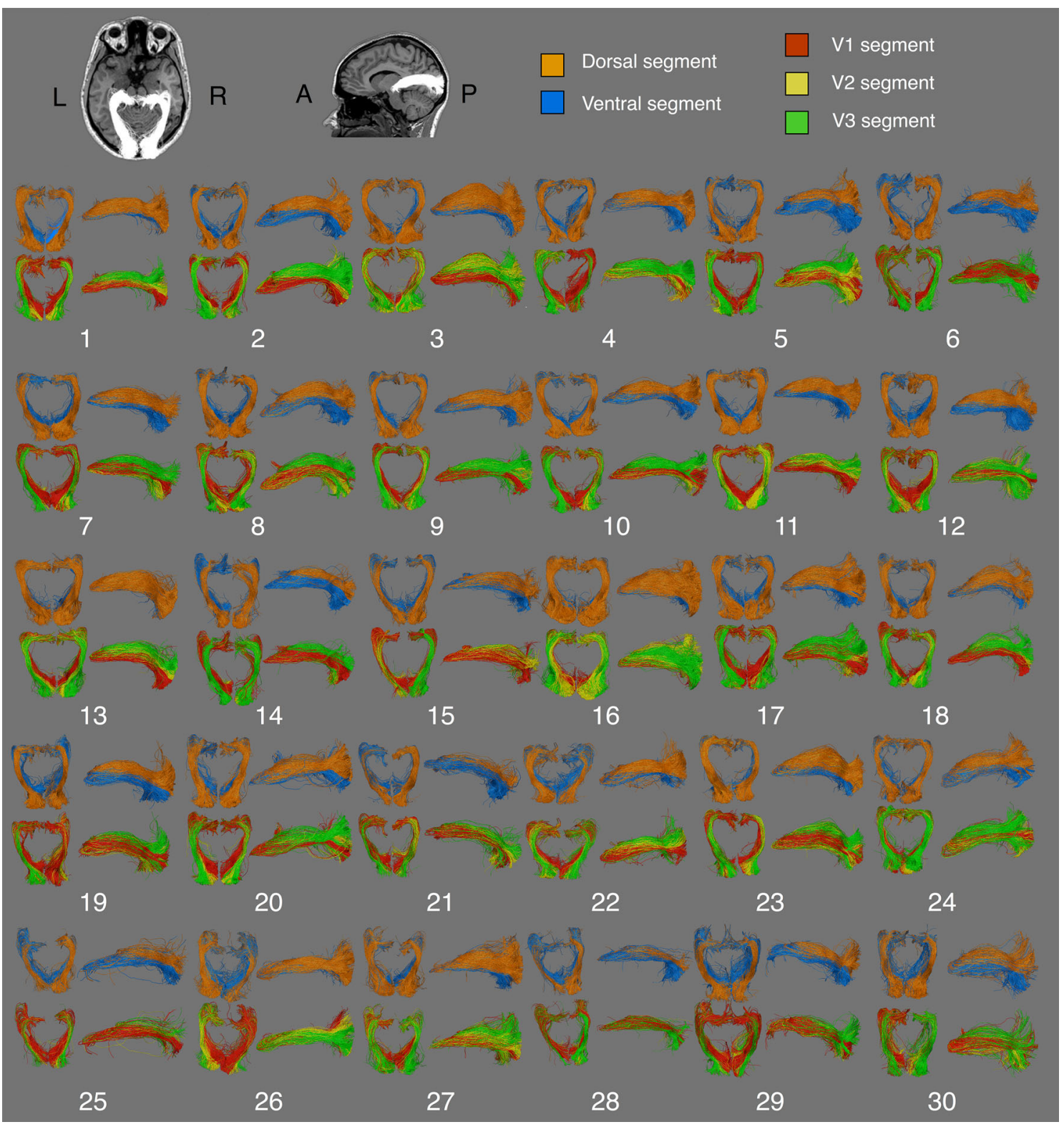

Fig. 6 Two forms of segmentation for the optic radiation are presented in thirty participants; visual field-based segmentation, yielding dorsal and ventral projections and hierarchy-based segmentation, yielding $V 1, V 2$ and $V 3$ projections. Two views are presented, an axial view from a superior vantage point, and a leftsided sagittal view from a lateral vantage point

\section{Appendix 2}

See Fig. 7. 




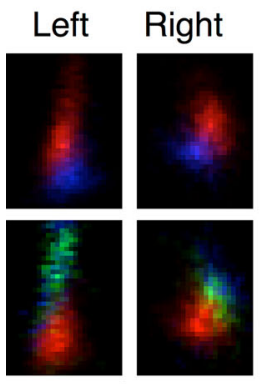

1


13
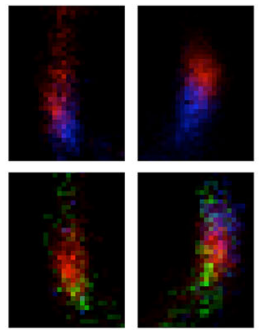

19
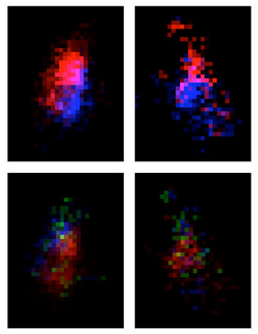

25

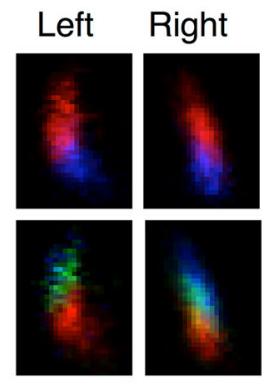

2

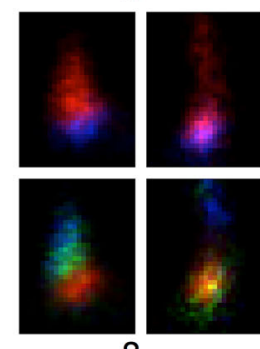

8

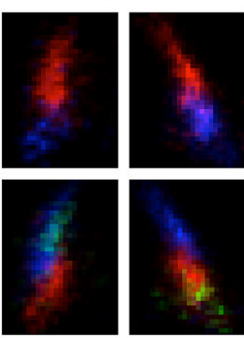

14
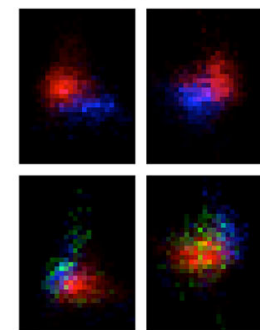

20


26

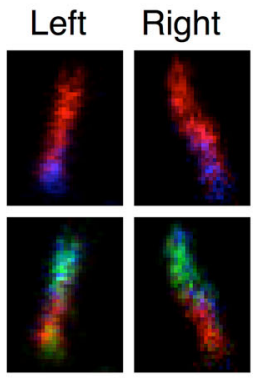

3

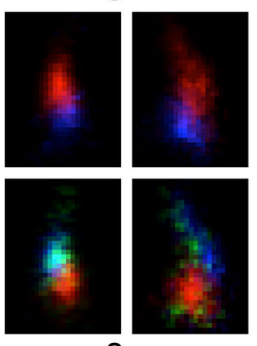

9



15
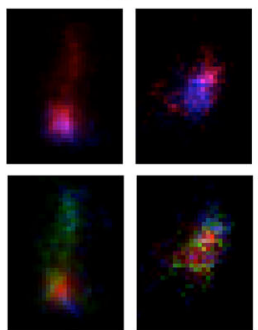

21
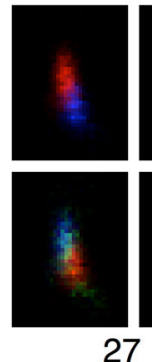

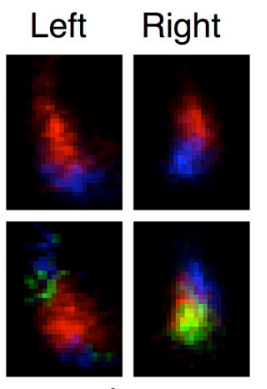

4

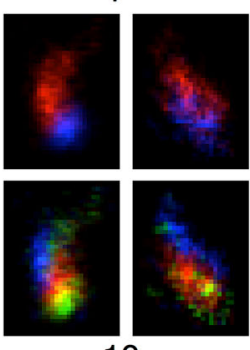

10

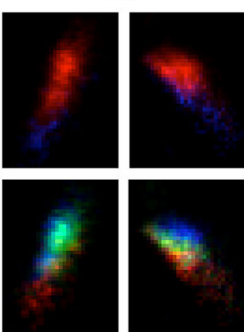

16


22
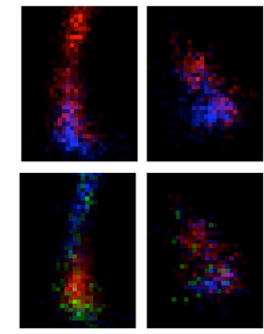

28



5

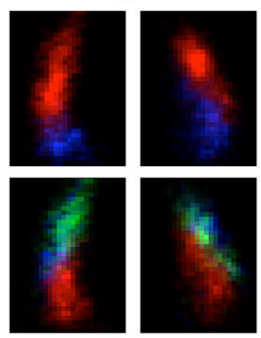

11

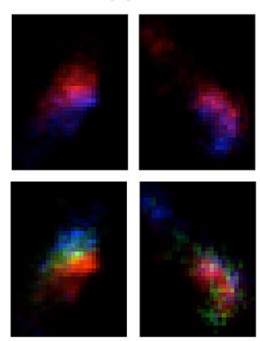

17
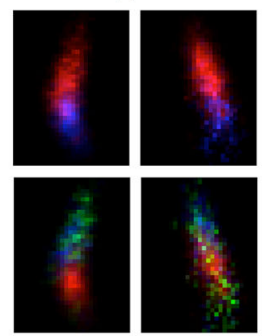

23
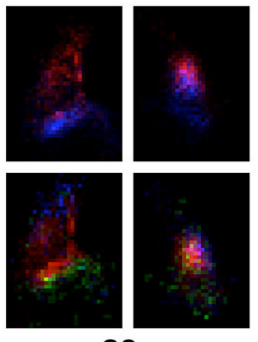

29

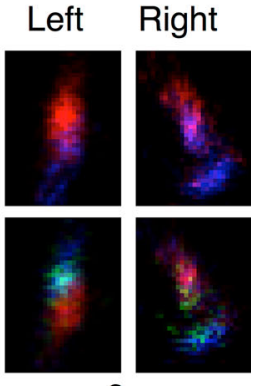

6

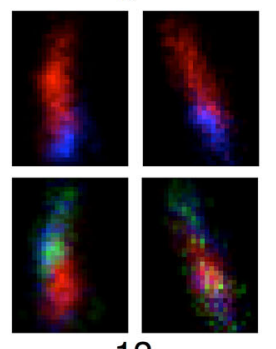

12

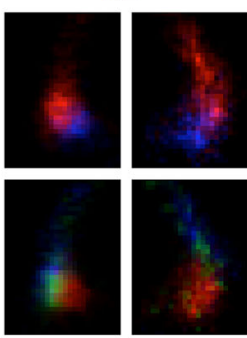

18
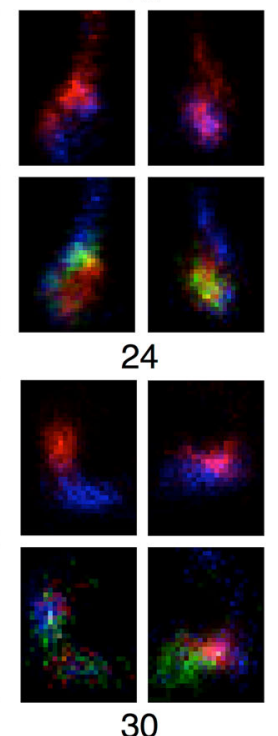
4Fig. 7 Visitation maps of optic radiation tractography in all thirty participants tested. All maps sampled at a single coronal slice immediately posterior to the lateral ventricle. Visual field-based segmentation shows the superior-inferior division of streamlines, in red and blue. Hierarchy-based segmentation discriminates streamlines into dorso-lateral nesting compartments, in red, green and blue

\section{References}

Barton JJS, Hefter R, Chang B, Schomer D, Drislane F (2005) The field defects of anterior temporal lobectomy: a quantitative reassessment of Meyer's loop. Brain 128:2123-2133

Basser PJ, Pajevic S, Pierpaoli C, Duda J, Aldroubi A (2000) In vivo fiber tractography using DT-MRI data. Magn Reson Med 44:625-632

Behrens TEJ, Woolrich MW, Jenkinson M, Johansen-Berg H, Nunes RG, Clare S et al (2003) Characterization and propagation of uncertainty in diffusion-weighted MR imaging. Magn Reson Med 50:1077-1088

Benevento LA, Standage GP (1982) Demonstration of lack of dorsal lateral geniculate nucleus input to extrastriate areas MT and visual 2 in the macaque monkey. Brain Res 252:161-166

Benevento LA, Yoshida K (1981) The afferent and efferent organization of the lateral geniculo-prestriate pathways in the macaque monkey. J Comp Neurol 203:455-474

Brainard DH (1997) The psychophysics toolbox. Spat Vis 10:433-436

Bridge H, Cowey A, Ragge N, Watkins K (2009) Imaging studies in congenital anophthalmia reveal preservation of brain architecture in 'visual' cortex. Brain 132:3467-3480

Bullier J, Kennedy H (1983) Projection of the lateral geniculate nucleus onto cortical area V2 in the macaque monkey. Exp Brain Res 53:168-172

Bürgel U, Schormann T, Schleicher A, Zilles K (1999) Mapping of histologically identified long fiber tracts in human cerebral hemispheres to the MRI volume of a reference brain: position and spatial variability of the optic radiation. Neuroimage 10:489-499

Catani M, Jones DK, Donato R, ffytche DH (2003) Occipito-temporal connections in the human brain. Brain 126:2093-2107

Chen W, Zhu XH, Thulborn KR, Ugurbil K (1999) Retinotopic mapping of lateral geniculate nucleus in humans using functional magnetic resonance imaging. Proc Natl Acad Sci USA 96:2430-2434

Clatworthy PL, Williams GB, Acosta-Cabronero J, Jones SP, Harding SG, Johansen-Berg H, Baron JC (2010) Probabilistic tractography of the optic radiations - an automated method and anatomical validation. Neuroimage 49:2001-2012

Daga P, Winston G, Modat M, White M, Mancini L, Cardoso MJ et al (2012) Accurate localization of optic radiation during neurosurgery in an interventional MRI suite. IEEE Trans Med Imaging 31:882-891

Dale AM, Fischl B, Sereno MI (1999) Cortical surface-based analysis-I: segmentation and surface reconstruction. Neuroimage 9:179-194

DeYoe EA, Carman GJ, Bandettini P, Glickman S, Wieser J, Cox R et al (1996) Mapping striate and extrastriate visual areas in human cerebral cortex. Proc Natl Acad Sci USA 93:2382-2386

Dougherty RF, Ben-Shachar M, Bammer R, Brewer AA, Wandell BA (2005) Functional organization of human occipital-callosal fiber tracts. Proc Natl Acad Sci USA 102:7350-7355

Dumoulin SO, Wandell BA (2008) Population receptive field estimates in human visual cortex. Neuroimage 39:647-660
Ebeling U, Reulen HJ (1988) Neurosurgical topography of the optic radiation in the temporal lobe. Acta Neurochir 92:29-36

El-Rafei A, Engelhorn T, Wärntges S, Dörfler A, Hornegger J, Michelson G (2011) A framework for voxel-based morphometric analysis of the optic radiation using diffusion tensor imaging in glaucoma. Magn Reson Imaging 29:1076-1087

Engel SA, Glover GH, Wandell BA (1997) Retinotopic organization in human visual cortex and the spatial precision of functional MRI. Cereb Cortex 7:181-192

Felleman DJ, Van Essen DC (1991) Distributed hierarchical processing in the primate cerebral cortex. Cereb Cortex 1:1-47

Fischl B, Sereno MI, Dale AM (1999) Cortical surface-based analysis. II: inflation, flattening, and a surface-based coordinate system. Neuroimage 9:195-207

Fries W (1981) The projection from the lateral geniculate nucleus to the prestriate cortex of the macaque monkey. Proc R Soc B 213:73-80

Friston KJ, Frith CD, Turner R, Frackowiak RS (1995) Characterizing evoked hemodynamics with fMRI. Neuroimage 2:157-165

Fujita N, Tanaka H, Takanashi M, Hirabuki N, Abe K, Yoshimura H, Nakamura $H$ (2001) Lateral geniculate nucleus: anatomic and functional identification by use of MR imaging. Am J Neuroradiol 22:1719-1726

Garey LJ, Powell TP (1971) An experimental study of the termination of the lateral geniculo-cortical pathway in the cat and monkey. Proc R Soc B 179:41-63

Groppo M, Ricci D, Bassi L, Merchant N, Doria V, Arichi T et al (2012) Development of the optic radiations and visual function after premature birth. Cortex (Advance online publication) [Epub ahead of print]

Hofer S, Karaus A, Frahm J (2010) Reconstruction and dissection of the entire human visual pathway using diffusion tensor MRI. Front Neuroanat 4:15

Kennedy H, Bullier J (1985) A double-labeling investigation of the afferent connectivity to cortical areas $\mathrm{V} 1$ and $\mathrm{V} 2$ of the macaque monkey. J Neurosci 5:2815-2830

Kim M, Ducros M, Carlson T, Ronen I, He S, Ugurbil K, Kim DS (2006) Anatomical correlates of the functional organization in the human occipitotemporal cortex. Magn Reson Imaging 24:583-590

Kolbe S, Bajraszewski C, Chapman C, Nguyen T, Mitchell P, Paine $M$ et al (2011) Diffusion tensor imaging of the optic radiations after optic neuritis. Hum Brain Mapp 33:2047-2061

Kommerell G, Schmitt C, Kromeier M, Bach M (2003) Ocular prevalence versus ocular dominance. Vision Res 43:1397-1403

Lagarias JC, Reeds JA, Wright MH, Wright PE (1998) Convergence properties of the Nelder-Mead simplex method in low dimensions. SIAM J Optim 9:112-147

Maciewicz RJ (1975) Thalamic afferents to areas 17, 18 and 19 of cat cortex traced with horseradish peroxidase. Brain Res 84:308-312

Manger PR, Rosa MGP (2005) Visual thalamocortical projections in the flying fox: parallel pathways to striate and extrastriate areas. Neuroscience 130:497-511

Oldfield RC (1971) The assessment and analysis of handedness: the Edinburgh inventory. Neuropsychologia 9:97-113

Párraga RG, Ribas GC, Welling LC, Alves RV, de Oliveira E (2012) Microsurgical anatomy of the optic radiation and related fibers in 3-dimensional images. Neurosurgery 71:160-172

Pelli DGD (1997) The VideoToolbox software for visual psychophysics: transforming numbers into movies. Spat Vis 10:437-442

Peltier J, Travers N, Destrieux C, Velut S (2006) Optic radiations: a microsurgical anatomical study. J Neurosurg 105:294-300

Peuskens D, van Loon J, Van Calenbergh F, van den Bergh R, Goffin J, Plets C (2004) Anatomy of the anterior temporal lobe and the 
frontotemporal region demonstrated by fiber dissection. Neurosurgery 55:1174-1184

Powell HWR, Parker GJM, Alexander DC, Symms MR, Boulby PA, Wheeler-Kingshott CAM et al (2005) MR tractography predicts visual field defects following temporal lobe resection. Neurology 65:596-599

Pujari VB, Jimbo H, Dange N, Shah A, Singh S, Goel A (2008) Fiber dissection of the visual pathways: analysis of the relationship of optic radiations to lateral ventricle: a cadaveric study. Neurol India 56:133-137

Reid RC, Alonso JM (1995) Specificity of monosynaptic connections from thalamus to visual cortex. Nature 378:281-284

Rice ML, Leske DA, Smestad CE, Holmes JM (2008) Results of ocular dominance testing depend on assessment method. J AAPOS 12:365-369

Rubino PA, Rhoton AL Jr, Tong X, de Oliveira E (2005) Threedimensional relationships of the optic radiation. Neurosurgery 57:219-227

Saenz M, Fine I (2010) Topographic organization of V1 projections through the corpus callosum in humans. Neuroimage 52:1224-1229

Schneider KA, Richter MC, Kastner S (2004) Retinotopic organization and functional subdivisions of the human lateral geniculate nucleus: a high-resolution functional magnetic resonance imaging study. J Neurosci 24:8975-8985

Seijas O, Gómez de Liaño P, Gómez de Liaño R, Roberts CJ, Piedrahita E, Diaz E (2007) Ocular dominance diagnosis and its influence in monovision. Am J Ophthalmol 144:209-216

Sereno MI, Dale AM, Reppas JB, Kwong KK, Belliveau JW, Brady TJ et al (1995) Borders of multiple visual areas in humans revealed by functional magnetic resonance imaging. Science 268:889-893

Sherbondy AJ, Dougherty RF, Napel S, Wandell BA (2008) Identifying the human optic radiation using diffusion imaging and fiber tractography. $\mathrm{J}$ Vis $8: 1-11$

Sincoff EH, Tan Y, Abdulrauf SI (2004) White matter fiber dissection of the optic radiations of the temporal lobe and implications for surgical approaches to the temporal horn. J Neurosurg 101:739-746

Tootell RB, Hadjikhani NK, Vanduffel W, Liu AK, Mendola JD, Sereno MI, Dale AM (1998) Functional analysis of primary visual cortex (V1) in humans. Proc Natl Acad Sci USA 95:811-817

Tournier JD, Calamante F, Connelly A (2012) MRtrix: diffusion tractography in crossing fiber regions. Int $\mathrm{J}$ Imaging Syst Technol 22:53-66

Van Essen DC, Maunsell JHR (1983) Hierarchical organization and functional streams in the visual cortex. Trends Neurosci 6:370-375

Wahler-Lück M, Schütz T, Kretschmann HJ (1991) A new anatomical representation of the human visual pathways. Graefes Arch Clin Exp Ophthalmol 229:201-205

Wandell BA, Winawer J (2011) Imaging retinotopic maps in the human brain. Vision Res 51:718-737

Weinberg DR, Luchins DJ, Morihisa J, Wyatt RJ (1982) Asymmetrical volumes of the right and left frontal and occipital regions of the human brain. Ann Neurol 11:97-100

Winston GP, Yogarajah M, Symms MR, McEvoy AW, Micallef C, Duncan JS (2011) Diffusion tensor imaging tractography to visualize the relationship of the optic radiation to epileptogenic lesions prior to neurosurgery. Epilepsia 52:1430-1438

Winston GP, Daga P, Stretton J, Modat M, Symms MR, McEvoy AW et al (2012) Optic radiation tractography and vision in anterior temporal lobe resection. Ann Neurol 71:334-341

Yan T, Jin F, Wu J (2009) Correlated size variations measured in human visual cortex V1/V2/V3 with functional MRI. Lect Notes Comput Sci 5819:36-44

Yogarajah M, Focke NK, Bonelli S, Cercignani M, Acheson J, Parker GJM et al (2009) Defining Meyer's loop-temporal lobe resections, visual field deficits and diffusion tensor tractography. Brain 132:1656-1668

Yoshida K, Benevento LA (1981) The projection from the dorsal lateral geniculate-nucleus of the thalamus to extrastriate visual association cortex in the macaque monkey. Neurosci Lett 22:103-108

Yukie M, Iwai E (1981) Direct projection from the dorsal lateral geniculate nucleus to the prestriate cortex in macaque monkeys. J Comp Neurol 201:81-97 